\title{
Fermi Level Position in GaMnAs - a Thermoelectric Study
}

\author{
V. Osinniy ${ }^{a}$, A. JęDrzejczaK ${ }^{a}$, M. ArCiszeWska ${ }^{a}$, \\ W. Dobrowolski ${ }^{a}$, T. StORY ${ }^{a}$ AND J. SADOWSKI ${ }^{b, a}$ \\ ${ }^{a}$ Institute of Physics, Polish Academy of Sciences \\ Al. Lotników 32/46, 02-668 Warsaw, Poland \\ ${ }^{b}$ MAX-lab, Lund University, 22100 Lund, Sweden
}

\begin{abstract}
Thermoelectric power was studied in the temperature range $100 \leq$ $T \leq 300 \mathrm{~K}$ in $0.3-1 \mu \mathrm{m}$ thick ferromagnetic $\mathrm{Ga}_{1-x} \mathrm{Mn}_{x}$ As epitaxial layers $(0.015 \leq x \leq 0.06)$ in order to determine Fermi energy $E_{\mathrm{F}}$ and carrier concentration $p$. For $0.015 \leq x \leq 0.05$, at $T=273 \mathrm{~K}$ we find $E_{\mathrm{F}}=275 \pm 50 \mathrm{meV}$ and $p=(2.5 \pm 0.5) \times 10^{20} \mathrm{~cm}^{-3}$ (approximately Mn content independent). For $x=0.06$, the Fermi energy decreases by about $100 \mathrm{meV}$ with the corresponding reduction of hole concentration to $p=1.2 \times 10^{20} \mathrm{~cm}^{-3}$. At $T=120 \mathrm{~K}$, these parameters vary between $E_{\mathrm{F}}=380 \mathrm{meV}$ and $p=3.5 \times 10^{20} \mathrm{~cm}^{-3}$ for $x=0.015$ to $E_{\mathrm{F}}=110 \mathrm{meV}$ and $p=5 \times 10^{19} \mathrm{~cm}^{-3}$ for $x=0.06$.
\end{abstract}

PACS numbers: $75.20 . \mathrm{Ck}, 75.30$.Et, 73.50.--h

\section{Introduction}

Ferromagnetism in $\mathrm{Ga}_{1-x} \mathrm{Mn}_{x}$ As is closely related to the electronic properties of this material. Particularly important is the presence of very high $(p \approx$ $10^{20}-10^{21} \mathrm{~cm}^{-3}$ ) concentration of quasi-free or weakly bound holes [1-4]. In contrast to II-VI and IV-VI semimagnetic (diluted magnetic) semiconductors with Mn ions, in GaMnAs and in InMnAs, Mn forms an electrically active acceptor center providing both the local magnetic moments and the required high concentration of carriers [5]. It is also well known that to obtain GaMnAs layers with Mn content of few atomic percents the, so-called, low-temperature molecular beam epitaxy (LT-MBE) has to be employed resulting in a creation of a very high (of the order of $10^{20} \mathrm{~cm}^{-3}$ ) concentration of native defects. These two factors make GaMnAs a material disordered with respect to both magnetic and electronic subsystem. Although the determination of such basic electronic parameters 
of GaMnAs as carrier concentration $p$ or Fermi energy $E_{\mathrm{F}}$ is of primary importance for the control of magnetic properties of this material, it is also a challenging experimental task. In particular, the use of standard Hall effect measurements is seriously complicated due to the large contribution of anomalous Hall effect and a large magnetoresistance. Recently, to firmly establish the concentration of carriers in GaMnAs, the Hall effect measurements were performed at the extreme conditions of very low temperatures (down to $50 \mathrm{mK}$ ) and very high magnetic fields (up to $27 \mathrm{~T}$ ) [6].

In this work, we examine the electronic properties of the epitaxial layers of GaMnAs using standard thermoelectric power $\alpha$ measurements, which proved to be a very efficient technique in the studies of strongly disordered condensed materials, like heavily doped or compensated semiconductors, and amorphous or liquid materials [7]. Our goal is, in particular, to verify whether the electronic parameters of GaMnAs such as the Fermi energy and the carrier concentration can be efficiently determined from the analysis of the thermoelectric power. In this work, we limit ourselves to the temperature range $T \geq 100 \mathrm{~K}$, i.e., to the paramagnetic phase of GaMnAs.

\section{Experimental results and discussion}

We studied thick $(0.3-1 \mu \mathrm{m})$ layers of $\mathrm{Ga}_{1-x} \mathrm{Mn}_{x} \mathrm{As}(0.01 \leq x \leq 0.06)$ grown by low-temperature MBE on GaAs (100) semi-insulating substrates with $0.1-0.2 \mu \mathrm{m}$ thick GaAs buffer layer grown by MBE at standard (high-temperature) conditions [8]. The thin layer between the GaMnAs and the buffer layer is the $4 \mathrm{~nm}$ thick LT-MBE GaAs layer (see Fig. 1). In both thermoelectric and magnetic measurements we used the layers of the typical area of $8 \times 4 \mathrm{~mm}^{2}$. For magnetic characterization of our layers, we carried out the measurements of the temperature dependence $(4.5 \leq T \leq 100 \mathrm{~K})$ of the ac magnetic susceptibility $\chi$ applying the ac magnetic fields of 5 Oe at a frequency of $625 \mathrm{~Hz}$. All the layers studied by us undergo ferromagnetic transition as evidenced by a strong sharp increase in the magnetic susceptibility. The ferromagnetic Curie temperature $T_{\mathrm{C}}$ is identified as the temperature corresponding to the maximum at the $\chi(T)$ dependence. For some layers, the initial sharp increase in magnetic susceptibility takes place at temperature $T_{\mathrm{C}}^{\mathrm{up}}$ considerably higher than $T_{\mathrm{C}}$. In our plot of the Mn composition dependence of $T_{\mathrm{C}}$ presented in Fig. 2, we show both $T_{\mathrm{C}}$ and $T_{\mathrm{C}}^{\mathrm{up}}$. The difference between these parameters might originate from the non-perfect homogeneity of the samples either in the distribution of magnetic ions or in hole concentration. For comparison, in Fig. 2 we also present the experimental data obtained from magnetization measurements performed for other samples grown with the same MBE facility in Lund [9]. The solid line indicates the record-high values as observed by Sendai group [1]. The differences are likely to stem from the variations of the conducting hole concentration - a parameter strongly dependent on the technological factors such as, e.g., the concentration of compensating donor centers. 


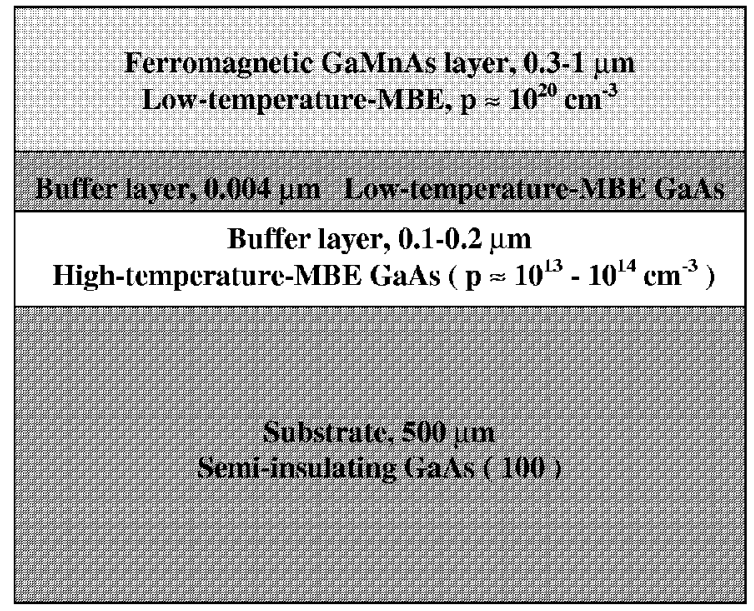

Fig. 1. The scheme of GaMnAs-GaAs heterostructures studied in this work. The thin layer (4 $\mathrm{nm}$ ) between GaMnAs layer and GaAs buffer is the GaAs layer grown at low-temperature MBE conditions.

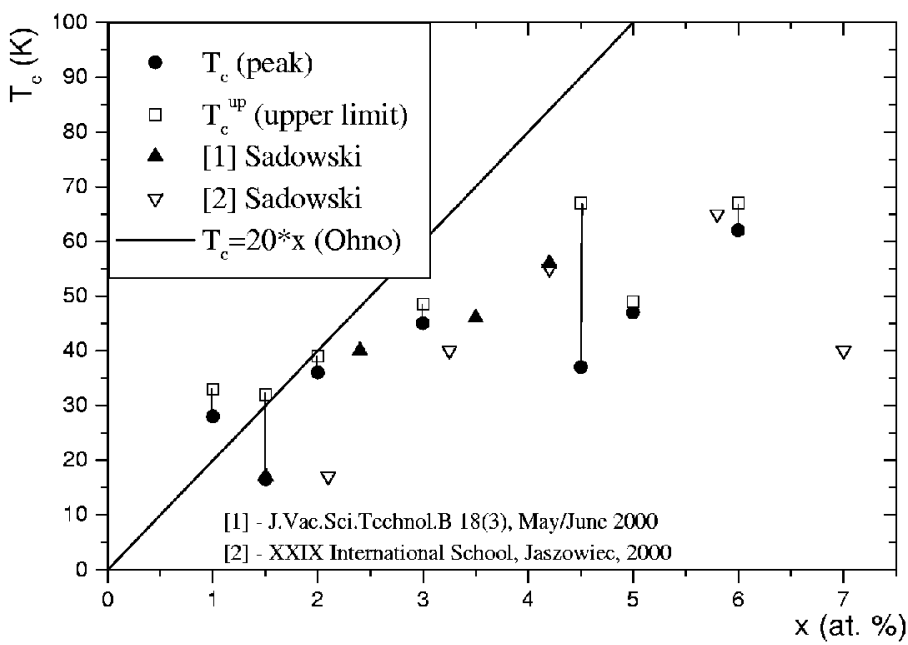

Fig. 2. The composition dependence of the ferromagnetic Curie temperature of $\mathrm{Ga}_{1-x} \mathrm{Mn}_{x}$ As. The solid line shows the dependence observed by Ohno for layers with the highest Curie temperatures. Triangles - the data obtained from magnetization measurements for layers grown by Sadowski.

The measurements of the thermoelectric power of $\mathrm{Ga}_{1-x} \mathrm{Mn}_{x} \mathrm{As}(0.015 \leq$ $x \leq 0.06$ ) layers were performed in the temperature range $T=100-300 \mathrm{~K}$. The resistance of GaMnAs layer with 1at.\% of Mn was too high for our experimental setup. The temperature difference (typically of $0.5 \mathrm{~K}$ ) was monitored with fine 
copper-constantan thermocouples attached to the layer from the top using silver conducting paste. The same paste was used to attach separate copper leads for thermoelectric voltage measurements. For each layer we performed the measurements at least twice with a change of the sign of the temperature gradient along the layer. The temperature dependence of thermoelectric power of GaMnAs is presented in Fig. 3.

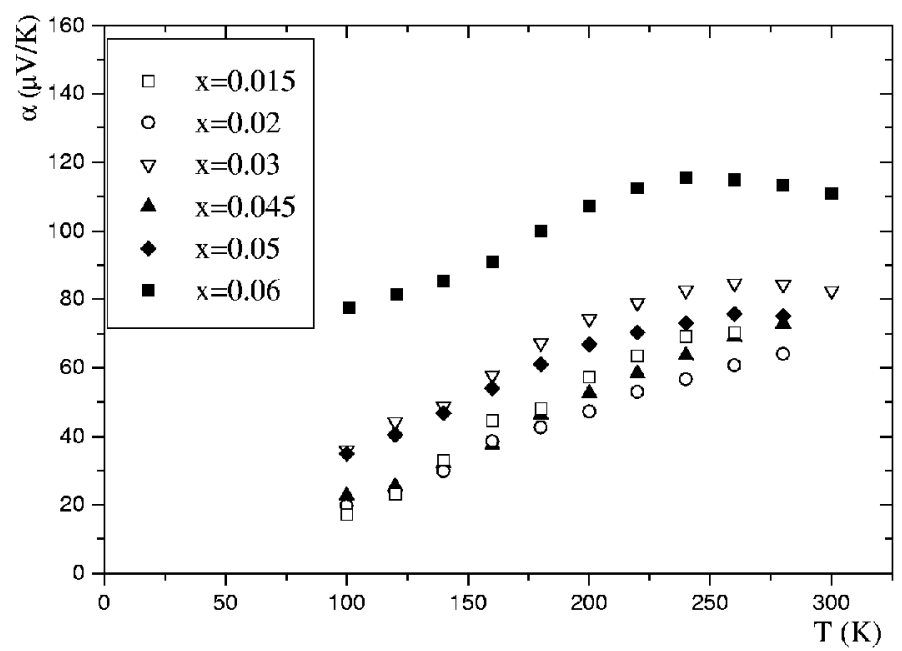

Fig. 3. The temperature dependence of the thermoelectric power in $\mathrm{Ga}_{1-x} \mathrm{Mn}_{x} \mathrm{As}$ epilayers.

For quantitative analysis of our thermoelectric power measurements, we have to analyze the role of GaAs buffer layer which, apart from the GaMnAs layer, is the second conducting layer in the heterostructure. The GaAs buffer was grown at standard (high-temperature) MBE conditions and no intentional doping was attempted. This layer is usually $p$-type with carrier concentration $p \approx 10^{13}-10^{14} \mathrm{~cm}^{-3}$. The thermoelectric parameters of a composite layered structure are calculated, e.g., in Ref. [10]. The experimentally measured "effective" thermoelectric power of the two-conducting-layer system, $\alpha_{\exp }$, is given by the expression

$$
\alpha_{\exp }=\frac{\alpha_{\mathrm{L}} \sigma_{\mathrm{L}} d_{\mathrm{L}}+\alpha_{\mathrm{B}} \sigma_{\mathrm{B}} d_{\mathrm{B}}}{\sigma_{\mathrm{L}} \mathrm{d}_{\mathrm{L}}+\sigma_{\mathrm{B}} d_{\mathrm{B}}} .
$$

Here $\alpha_{\mathrm{L}, \mathrm{B}}, \sigma_{\mathrm{L}, \mathrm{B}}$, and $d_{\mathrm{L}, \mathrm{B}}$ refer to the thermoelectric power, conductivity, and thickness of GaMnAs layer (L) and buffer layer (B), respectively.

Since for our structures typically $\sigma_{\mathrm{L}} d_{\mathrm{L}} / \sigma_{\mathrm{B}} d_{\mathrm{B}} \approx 10^{3}$, in practice one has $\alpha_{\exp } \approx \alpha_{\mathrm{L}}$. The low-temperature MBE GaAs layer present on top of the buffer layer is more than 100 times thinner than the other layers. Therefore, in our analysis we neglected the possible contribution of this layer. 
In the temperature range studied, the thermoelectric power in all GaMnAs layers investigated by us shows $p$-type conductivity. At lower temperatures, the temperature dependence of the thermoelectric power is practically linear with the tendency to decrease the slope while approaching the room temperature. This behavior is qualitatively similar to the temperature dependence of thermoelectric power observed in other strongly $p$-type doped semiconductor alloys like, e.g., $\mathrm{Si}_{1-x} \mathrm{Ge}_{x}$ :B with hole concentrations up to $p=10^{21} \mathrm{~cm}^{-3}$ [11]. One can consider this observation as an experimental evidence of the existence in GaMnAs of a degenerate conducting hole gas. We observe only minor differences between the thermoelectric parameters of GaMnAs epilayers with different Mn content, which is in contrast with the well-known metallic character of conductivity in GaMnAs with $0.03 \leq x \leq 0.05$ and its semiconducting character for both $x<0.03$ and $x>0.05$. It suggests that the activation energies for conductivity and for conducting hole concentration are different. The last one is known to determine the temperature dependence of thermoelectric power in disordered materials [7]. The solution of this issue requires an extension of thermoelectric measurements down to liquid helium temperatures and will be presented in a separate paper. Our preliminary data indicate that the quasi-linear temperature dependence is observed down to about $50 \mathrm{~K}$. Below this temperature, an up-turn of $\alpha(T)$ dependence is observed — the effect well known in disordered electronic systems [7].

To quantitatively analyze our experimental data, we apply standard expressions for thermoelectric power in doped semiconductors (for arbitrary degree of degeneracy) with a parabolic energy dispersion relation. To calculate the Fermi energy one has to specify the scattering parameter $r$. However, the role of different scattering mechanisms in GaMnAs is not well established. The temperature dependence of resistivity near to the Curie temperature is well described by the spin-dependent scattering on the fluctuations of magnetic subsystem of $\mathrm{Mn}$ ions $[1,2]$. However, the carrier mobilities encountered in GaMnAs are extremely low $\mu=1-10 \mathrm{~cm}^{2} /(\mathrm{V} \mathrm{s})$, which is related to the very high concentration of defects inherent to GaMnAs due to the presence of electrically active Mn ions and the low-temperature mode of growth introducing a high concentration of compensating donor centers $[12,13]$. If various scattering mechanisms contribute to charge transport in a semiconductor, the effective scattering parameter which enters the expression for thermoelectric power is primarily determined by the mechanism yielding the smallest mobility. Therefore, in our analysis we adopt a simple model assuming that the dominant scattering mechanism is due to the scattering on ionized impurities. The Fermi energies obtained within the above described approach are presented in Fig. 4a. As expected for a degenerate conducting hole system, we find the Fermi level located rather deep below the top of the valence band. To calculate the conducting hole concentration, additionally, the effective mass of density of states is required which we take equal to $m_{d}^{*}=0.5 m_{0}$ [1]. The carrier concentrations obtained from our thermoelectric power measurements are 

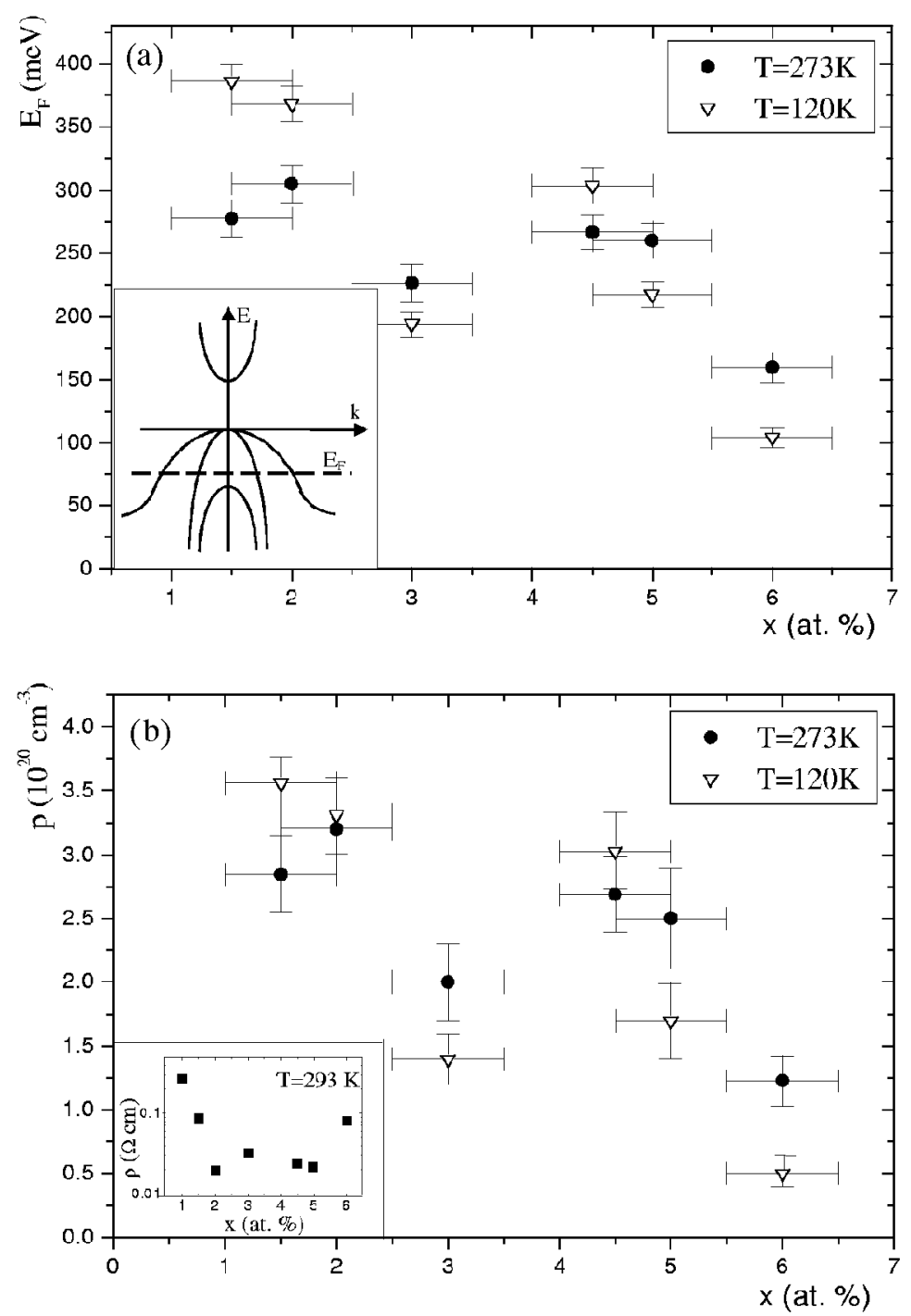

Fig. 4. The Fermi energy $E_{\mathrm{F}}$ (a) and the concentration of conducting holes $p$ (b) in $\mathrm{Ga}_{1-x} \mathrm{Mn}_{x}$ As as determined from thermoelectric power measurements. The inset in part (b) presents the dependence of the resistivity (at room temperature) of GaMnAs layers on Mn content.

presented in Fig. 4b. One has to note that in our analysis of thermoelectric power of $\mathrm{GaMnAs}$, the valence band is characterized by a single isotropic parameter $m_{d}^{*}$ - the effective mass of density of states. We neglected peculiarities related to: (1) the expected non-parabolicity and anisotropy of the valence band, and (2) the stress-induced removal of the degeneracy of the light- and heavy-hole bands, as discussed in detail by Dietl et al. in Ref. [4]. 
The Fermi energy and the hole concentration determined from our thermoelectric power measurements agree rather well with other experimental data and the established physical picture of GaMnAs. That somehow supports our choice of parameters and method of analysis. However, one has to underline that our quantitative conclusions are also based on the assumption that the standard expressions for thermoelectric power in doped semiconductors are valid for GaMnAs. At this stage of the analysis, we neglected the possible disorder-induced changes in the charge transport mechanisms or valence band structure as the current understanding of these effects in GaMnAs is rather limited.

\section{Conclusions}

Our thermoelectric measurements of GaMnAs epitaxial layers show that in the temperature range $100 \leq T \leq 300 \mathrm{~K}$ the thermoelectric power increases with increasing temperature in a quasi-linear way observed also in other strongly $p$-type doped semiconductors. The quantitative analysis was performed assuming the effective mass of density of states equal to $m_{d}^{*}=0.5 m_{0}$, the dominance of ionized impurity scattering, and the applicability of standard theory of thermoelectric effects in doped semiconductors to GaMnAs. At $T=273 \mathrm{~K}$, for $0.015 \leq x \leq 0.05$, we found that the Fermi level is located about $E_{\mathrm{F}} \approx 275 \mathrm{meV}$ below the top of the valence band, which corresponds to the hole concentration $p \approx 2.5 \times 10^{20} \mathrm{~cm}^{-3}$. For $x=0.06$ we observe the decrease in the Fermi energy by about $100 \mathrm{meV}$ with the corresponding twofold reduction of hole concentration. At $T=120 \mathrm{~K}$ the physical picture is qualitatively similar. The parameters obtained applying the simple thermoelectric method compare well with the hole concentration and the Fermi energy values calculated theoretically or determined in other experiments.

\section{Acknowledgments}

This work was partially supported by the State Committee for Scientific Research (Poland) project No. 2 P03B 15418 and within European Community program ICA1-CT-2000-70018 (Centre of Excellence CELDIS).

\section{References}

[1] H. Ohno, J. Magn. Magn. Mater. 200, 110 (1999).

[2] T. Dietl, H. Ohno, Physica E 9, 185 (2001).

[3] A. Van Esch, L. Van Bockstal, J. de Boek, G. Verbanck, A.S. Van Steenbergen, P.J. Wellmann, B. Grietens, R. Bogaerts, F. Herlach, G. Borghs, Phys. Rev. B 56, 13103 (1997).

[4] T. Dietl, H. Ohno, F. Matsukura, Phys. Rev. B 63, 195205 (2001); see also Phys. Rev. B 63, 054418 (2001). 
[5] A. Twardowski, Acta Phys. Pol. A 98, 203 (2000).

[6] T. Omiya, F. Matsukura, T. Dietl, Y. Ohno, T. Sakon, M. Motokawa, H. Ohno, Physica E 7, 976 (2000).

[7] N.F. Mott, E.A. Davis, Electron Processes in Non-Crystalline Materials, Clarendon Press, Oxford 1979.

[8] J. Sadowski, J.Z. Domagała, J. Bąk-Misiuk, S. Koleśnik, K. Świątek, J. Kanski, L. Ilver, Thin Solid Films 367, 165 (2000).

[9] J. Sadowski, J.Z. Domagała, J. Bąk-Misiuk, S. Koleśnik, M. Sawicki, K. Świątek, J. Kanski, L. Ilver, V. Strom, J. Vac. Sci. Technol. B 18, 1697 (2000).

[10] P.J. Lin-Chung, T.L. Reinecke, Phys. Rev. B 51, 13244 (1995).

[11] G.A. Slack, M.A. Hussain, J. Appl. Phys. 70, 2694 (1991).

[12] A. Oiwa, S. Katsumoto, A. Endo, M. Hirasawa, Y. Iye, H. Ohno, F. Matsukura, A. Shen, Y. Sugawara, Solid State Commun. 103, 209 (1997).

[13] F. Matsukura, H. Ohno, A. Shen, Y. Sugawara, Phys. Rev. B 57, R2037 (1998). 